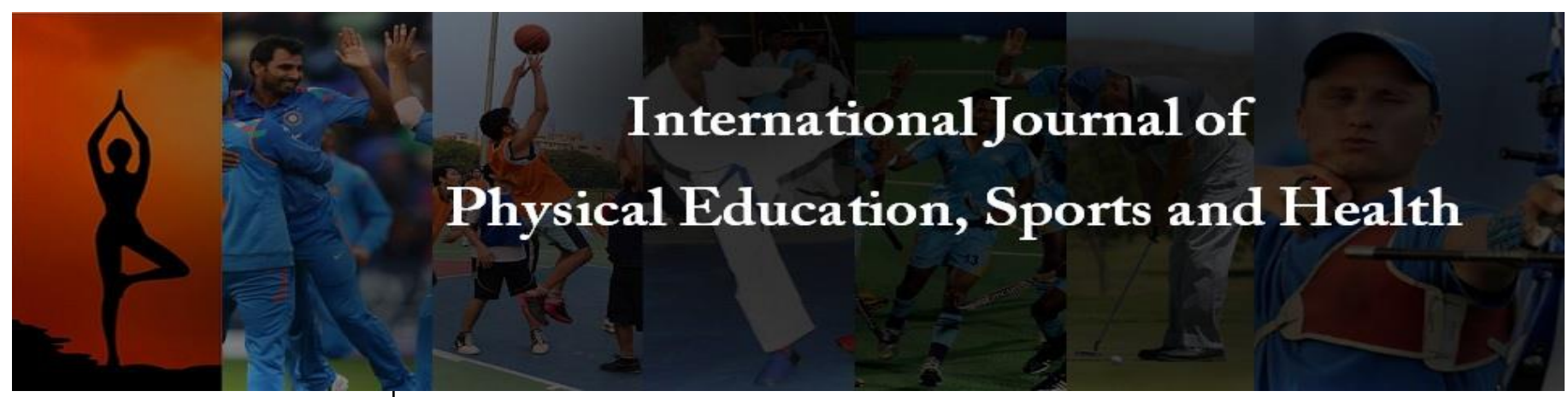

P-ISSN: 2394-1685

E-ISSN: 2394-1693

Impact Factor (ISRA): 5.38

IJPESH 2021; 8(3): 286-289

(C) 2021 IJPESH

www.kheljournal.com

Received: 10-03-2021

Accepted: 12-04-2021

\section{Aayushi Kamdar}

Ahmedabad Institute of

Physiotherapy, Gujarat

University, Ahmedabad,

Gujarat, India

\section{Hardini Prajapati}

Ahmedabad Institute of

Physiotherapy, Gujarat

University, Ahmedabad,

Gujarat, India
Corresponding Author: Aayushi Kamdar Ahmedabad Institute of Physiotherapy, Gujarat University, Ahmedabad, Gujarat, India

\section{Level of physical activity in rural and urban elementary school children: An observational study}

\author{
Aayushi Kamdar and Hardini Prajapati
}

DOI: https://doi.org/10.22271/kheljournal.2021.v8.i3e.2097

\section{Abstract}

Physical activity is influenced by various factors such as geographical background, socio-economic status, urban/rural dwelling etc. A better understanding of rural and urban prevalence in level of physical activity leads to targeted physical activity intervention. The sample consisted of 100 elementary school children; 50(urban) and 50(rural) locality of Ahmedabad city aged 8-14 years. Physical Activity Questionnaire for Children [PAQ-C] Gujarati version was used to assess various dimensions of physical activity. On PAQ-C score for Urban population, 14\%showed High Physical Activity; $16 \%$ showed Low Physical Activity; 70\% showed Moderate Physical Activity. PAQ-C score for Rural Population, 24\% showed High Physical Activity; 12\% showed Low Physical Activity; 64\% showed Moderate Physical Activity. Children living in rural areas are more physically active than urban though overall PAQ-C score displayed moderate activity. Boys displayed higher physical activity than girls in both urban and rural settings.

Keywords: Elementary school children, physical activity, PAQ-C questionnaire

\section{Introduction}

According to ACSM Guidelines "Physical Activity is defined as any bodily movement produced by the contraction of skeletal muscles that result in a substantial increase over resting energy expenditure." "1]. Physical activity in daily life can be categorized into occupational, sports, conditioning, household and other activities. The American Physical Activity Guidelines recommended that school-aged children and adolescents participate in at least 60 min of moderate to vigorous PA each day ${ }^{[2]}$. Elementary school children are at a very important age of their development. It becomes absolutely mandatory to inculcate play habits in their daily regimen. Cardiovascular fitness is also important because cardiovascular fitness is inversely associated with being overweight in children and adolescents.

In March 2020, the respiratory disease caused by the SARS-COV-2 virus, COVID-19, was declared a pandemic by the World Health Organization ${ }^{[3]}$. During the corona virus disease 2019 (COVID-19) pandemic, the government adopted containment measures to control the virus's spread, including limitations to the practice of Physical Activity (PA). The government promoted social distancing, closure of schools, suspension of any social event which promoted mass gathering etc as a means to control the viral spread. To further counter the spread of the virus, access to public parks, gardens, sports center, swimming pools, outdoor recreational centers etc were prohibited ${ }^{[4]}$. Such confinement rules led to increasingly restrictive practice of PA. Among the worst affected by such measures are children who couldn't engage in any kind of outdoor play activities. They no longer had access to school-based physical activities such as physical education, and walking to/from school ${ }^{[3]}$.

Covid-19 confinement measures have sent us into a whole new world of stagnancy and eroded resources to remain physically active. The core part of this population are children who are directly or indirectly affected. Moreover, physical inactivity has contributed to adverse health hazards particularly aiming at childhood obesity, muscle atrophy and decreased aerobic capacity.

Several studies have been done on the sedentary behavior of children. This represents a mass exposure of growing population to health risks like childhood obesity and juvenile 
hypertension. A better understanding of Rural and Urban prevalence of physical activity leads to targeted awareness interventions as well as policy initiatives at population level. Therefore, the purpose of this study is to find out the level of physical activity in rural and urban children of age 8-14 years. Given the potential risk of obesity, diabetes and other chronic diseases in children due to prolonged physical inactivity, information about the impact of COVID-19 pandemic on children's PA can inform immediate policy efforts during the pandemic.

\section{Materials and Methods}

A study was performed among 100 elementary school students of age 8-14 years including both male and female. 50 subjects were taken from rural locality and 50 were taken from urban locality. A structured questionnaire "Physical Activity Questionnaire for Older Children (PAQ-C)" was filled by the students and further Data Analysis was done in Microsoft Excel. Both male and female children from grade $4^{\text {th }}$ to $8^{\text {th }}$, knowing Gujarati language and willing to participate in the study were included. Children who reported to be sick on PAQ-C were excluded as it prevented them from doing any physical activity in the last week.

\subsection{Outcome Measure}

The Gujarati version of Physical Activity Questionnaire for Older Children (PAQ-C) was used to assess the Physical Activity level of children. PAQ-C is a self-administered, 7 day recall instrument. It was developed to assess general levels of physical activity throughout the elementary school year students. It is a 9-item self-report tool each scored on a 5 point scale. A score of 1 indicates low physical activity, whereas a score of 5 indicates high physical activity. The testretest reliability correlation coefficient is 0.94 which showed a strong correlation and a valid tool to measure the physical activity level of children ${ }^{[5]}$.

\subsection{Procedure}

Subjects were randomly selected on the basis of inclusion and exclusion criteria. They were asked to give a score from 1 to 5 on each item of the questionnaire. They filled the questionnaire on the basis of their recall of activity done in the last 7 days. The questionnaire took approximately 20 minutes to complete by each subject. Total score was found by calculating the mean of each item and then finding the overall summary activity score. A score of 1 indicated low physical activity and a score of 5 indicated high physical activity. Data analysis was done using Microsoft Excel.

\section{Results and Discussion}

The mean score of rural locality was found to be $2.728 \pm$ 0.755. The prevalence of Physical Activity in rural locality showed that $24 \%$ population had High Physical Activity levels, 64\% had moderate Physical Activity levels and 12\% had Low Physical Activity levels.

The mean score of urban locality was found to be $2.455 \pm$ 0.807 . The prevalence of physical activity in urban locality showed that $14 \%$ population had High Physical Activity levels, $70 \%$ had moderate physical activity levels and 16\% had Low Physical activity levels.

Rural girls had a mean score of $2.620 \pm 0.749$ whereas rural boys had a mean score of $2.793 \pm 0.777$. Urban girls had a mean score of $2.278 \pm 0.824$ whereas urban boys had a mean score of $2.583 \pm 0.783$.

Table 1: Mean age Group and PAQ-C score of Rural and Urban Localities

\begin{tabular}{|c|c|c|c|}
\hline Locality & N & Age(years) & PAQ-C Score \\
\hline Rural & 50 & $11.06 \pm 2.11$ & $2.728 \pm 0.755$ \\
\hline Urban & 50 & $10.92 \pm 2.10$ & $2.455 \pm 0.807$ \\
\hline
\end{tabular}

Table 2: PAQ-C Score of Girls and Boys from Rural and Urban

\begin{tabular}{|c|c|c|}
\hline Gender & N & PAQ-C Score \\
\hline Girls (rural) & 28 & $2.620 \pm 0.749$ \\
\hline Boys (rural) & 22 & $2.793 \pm 0.777$ \\
\hline Girls (urban) & 21 & $2.278 \pm 0.824$ \\
\hline Boys (urban) & 29 & $2.583 \pm 0.783$ \\
\hline
\end{tabular}

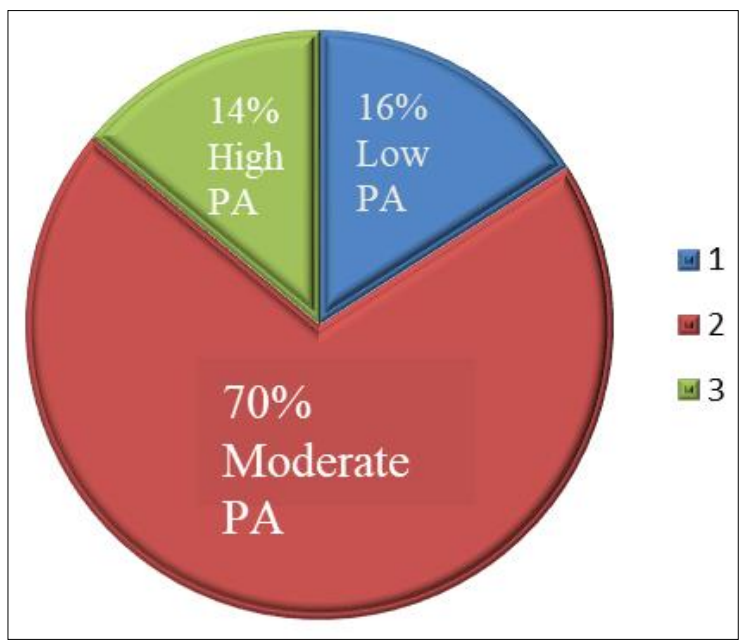

Graph 1: Showing prevalence of PA in urban population

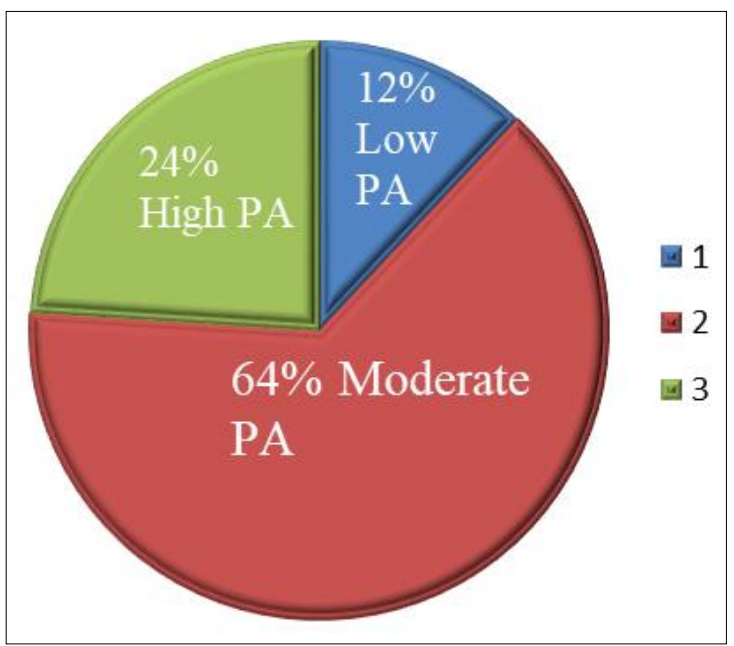

Graph 2: Showing prevalence of PA in rural population 


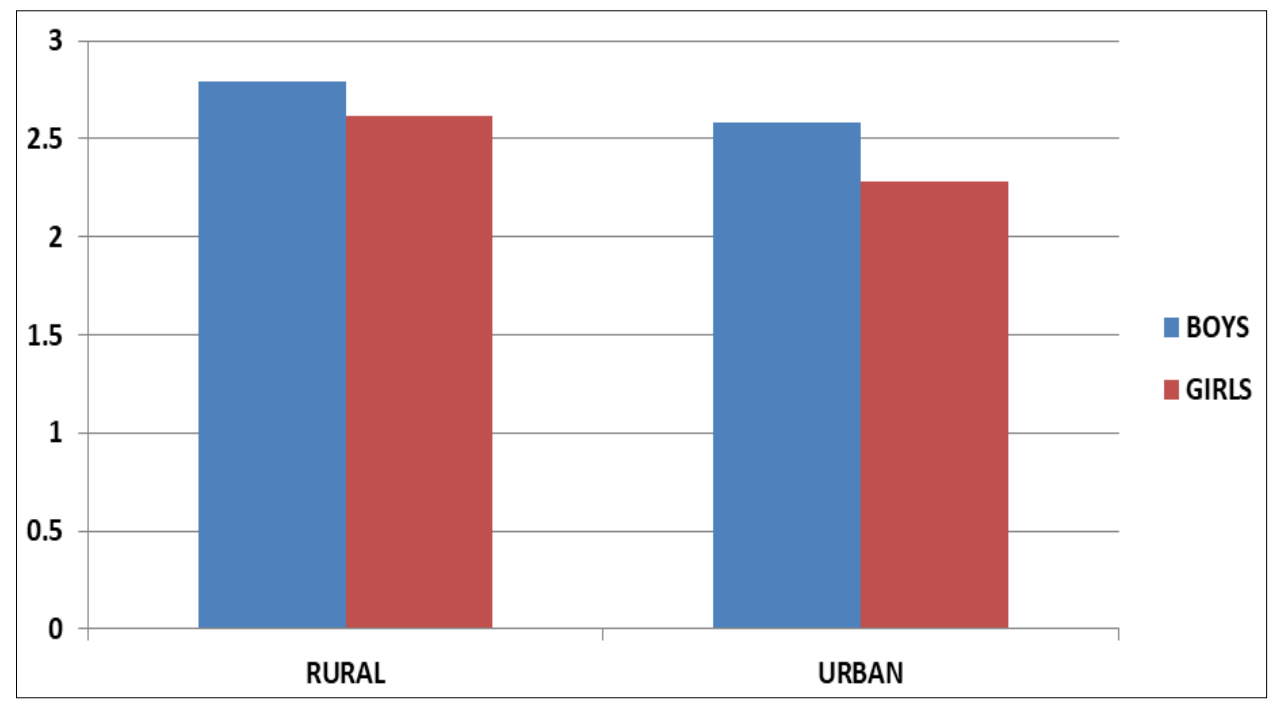

Graph 3: Showing Boys having higher physical activity than Girls in both Urban and Rural

There is certainly a close relationship between socioeconomic status, geographical variations and physical activity levels. It has been found that the overall score of PAQ-C of both the localities was moderate, though, rural children were found to be slightly more physically active than urban. Due to temporary closure of schools and adoption of online platform for education, children have been engaging lesser time in play activities and more on screen time. The abrupt reduction of PA induces several ill effects in the human body both in short term and long term. Firstly, the drastic decrement of PA leads to acute modifications such as atrophy and muscle mass reduction in a few days. It can also lead to failure to maintain body weight, increasing health risks. The sedentary approach adopted by children due to Covid-19 confinement induces adaptations that negatively affect the cardio respiratory fitness and metabolic profile.

Due to the benefits of PA on psycho-physiological human functions that in this critical period could be compromised, major institutions as the World Health Organization (WHO) and the American College of Sports Medicine (ACSM) developed guidelines for healthy people, recommending specific exercise programs and daily strategies to adopt during quarantine ${ }^{[4]}$.

Similar studies done in Turkey and Cyprus show that rural children are more physically active than urban ${ }^{[6,7]}$. This is inconsistent with the present study which shows that isolated confinement and online schooling has adversely affected both urban and rural children equally. The results indicate insufficient activity levels among urban children as compared to rural children though both populations displayed sedentary behaviours. Rural Mississippi youth also reported lower physical activity levels than a comparison national sample, although geographical settings for national sample were not specified ${ }^{[8]}$. In contrast, no differences in physical activity were reported in studies comparing youth of rural and urban residence in North Carolina, although differences were noted in obesity and cardiovascular risk factor profiles ${ }^{[8]}$. The inconsistent nature of these studies may be due to confounding by other variables.

The advantage of the present study is that differences in time and location of physical activity were explored by examining individual items on the PAQ-C. A study investigating intensity of physical activity found that boys reported more moderate and vigorous intensity physical activities than girls. This is consistent with the present study which showed that boys are shown to have higher level of physical activity than girls in both rural and urban localities. Physical activity levels in children from both locations were similar on weekends. The main advantage of the study was that geographical settings were included in the study which gave a better explanation of rural and urban prevalence. Providing physical activity after lunchtime as well as after the online classes can help break the chain of sedentary lifestyle approach which has found its way through this pandemic. Since physical inactivity is positively related to higher rate of morbidity and an increase of incidence of mortality, the scientific literature reinforced the importance of maintaining an active physical state, even during the current pandemic. In fact, it is widely recognized that PA induces positive effects on different psycho-physiological aspects at every stage of life. Interventions should be uniquely tailored according to the ecological settings of each population.

Moreover, our findings seem to suggest that a home-based setting is not favorable for PA. Since insufficiency of large spaces for PA could be an influential factor in reduction of PA, our further study aims to extend the analysis using multivariate models with respect to other variables of the questionnaire including home setting (e.g.: availability of outdoor space) ${ }^{[4]}$.

\section{Conclusion}

Children living in rural areas are more physically active than those residing in urban areas though majorly both portrayed moderate physical activity. The most significant finding related to this study shows that confinement due to pandemic increases screen time and decreases play activities. Based on our outcomes, we can determine that the current quarantine has negatively influenced and has greater impact on PA, especially children. For this reason, it is recommended to increase home-based training during quarantine. Furthermore, excessive training can also lead to musculoskeletal injuries, we suggest maintaining PA level closer to habitual and mild to moderate intensity and rather encouraging to follow play activities which children used to do at school.

\section{Acknowledgements}

Authors would like to thank the children who participated in the study.

\section{References}

1. Martin SB, Morrow JR, Jackson AW, Dunn AL. Variables related to meeting the CDC/ACSM physical 
activity guidelines. Medicine and science in sports and exercise 2000;32(12):2087-92.

2. Tucker JM, Welk GJ, Beyler NK. Physical activity in US adults: compliance with the physical activity guidelines for Americans. American journal of preventive medicine 2011;40(4):454-61.

3. Dunton GF, Do B, Wang SD. Early effects of the COVID-19 pandemic on physical activity and sedentary behavior in children living in the US. BMC Public Health 2020;20(1):1-3.

4. Giustino V, Parroco AM, Gennaro A, Musumeci G, Palma A, Battaglia G. Physical activity levels and related energy expenditure during COVID-19 quarantine among the Sicilian active population: a cross-sectional online survey study. Sustainability 2020;12(11):4356.

5. Belim ZK, Sheth M, Sharma S. Reliability and validity of Gujarati version of physical activity questionnaire for children. Int J Phys Educ Sports Health 2019;6:154-7.

6. Bathrellou E, Lazarou C, Panagiotakos DB, Sidossis LS. Physical activity patterns and sedentary behaviors of children from urban and rural areas of Cyprus. Central European journal of public health 2007;15(2):66-70.

7. Özdirenç M, Özcan A, Akin F, Gelecek N. Physical fitness in rural children compared with urban children in Turkey. Pediatrics international 2005;47(1):26-31.

8. Joens- Matre RR, Welk GJ, Calabro MA, Russell DW, Nicklay E, Hensley LD. Rural-urban differences in physical activity, physical fitness, and overweight prevalence of children. The Journal of rural health 2008;24(1):49-54.

9. Kisner C, Colby LA, Borstad J. Therapeutic exercise: foundations and techniques. Fa Davis 2017-18.

10. Kowalski KC, Crocker PR, Donen RM. The physical activity questionnaire for older children (PAQ-C) and adolescents (PAQ-A) manual. College of Kinesiology, University of Saskatchewan 2004;87(1):1-38.

11. Zenic N, Taiar R, Gilic B, Blazevic M, Maric D, Pojskic $\mathrm{H}$ et al. Levels and changes of physical activity in adolescents during the COVID-19 pandemic: contextualizing urban vs. rural living environment. Applied Sciences 2020;10(11):3997.

12. Loucaides CA, Chedzoy SM, Bennett N. Differences in physical activity levels between urban and rural school children in Cyprus. Health education research 2004;19(2):138-47.

13. Moore JB, Brinkley J, Crawford TW, Evenson KR, Brownson RC. Association of the built environment with physical activity and adiposity in rural and urban youth. Preventive medicine 2013;56(2):145-8.

14. Liu JH, Jones SJ, Sun H, Probst JC, Merchant AT, Cavicchia P. Diet, physical activity, and sedentary behaviors as risk factors for childhood obesity: an urban and rural comparison. Childhood Obesity (Formerly Obesity and Weight Management) 2012;8(5):440-8.

15. Khan BZ, Sheth M. Physical activity level and physical fitness parameters in physiotherapy students. Int J Physiother Res 2019;7(5):3247-51.

16. Kantanista A, Osiński W, Borowiec J, Tomczak M, KrólZielińska M. Body image, BMI, and physical activity in girls and boys aged 14-16 years. Body image 2015;15:403.

17. Patnode CD, Lytle LA, Erickson DJ, Sirard JR, BarrAnderson D, Story $M$. The relative influence of demographic, individual, social, and environmental factors on physical activity among boys and girls.
International Journal of Behavioural Nutrition and Physical Activity 2010;7(1):1-0.

18. Janz KF, Lutuchy EM, Wenthe P, Levy SM. Measuring activity in children and adolescents using self-report: PAQ-C and PAQ-A. Medicine and science in sports and exercise 2008;40(4):767-72.

19. Benítez- Porres J, López- Fernández I, Raya JF, Álvarez Carnero S, Alvero- Cruz JR, Álvarez Carnero E. Reliability and validity of the PAQ- C questionnaire to assess physical activity in children. Journal of School Health 2016;86(9):677-85.

20. Wang JJ, Baranowski T, Lau WP, Chen TA, Pitkethly AJ. Validation of the physical activity questionnaire for older children (PAQ-C) among Chinese children. Biomedical and Environmental Sciences 2016;29(3):17786. 